\title{
Cascade events at IceCube + DeepCore as a definitive constraint on the dark matter interpretation of the PAMELA and Fermi anomalies
}

\author{
Sourav K. Mandal, ${ }^{1,2}$ Matthew R. Buckley, ${ }^{3}$ Katherine Freese, ${ }^{4}$ Douglas Spolyar, ${ }^{5,6}$ and Hitoshi Murayama ${ }^{1,2,7}$ \\ ${ }^{1}$ Department of Physics, University of California, Berkeley, California 94720, USA \\ ${ }^{2} I P M U$, University of Tokyo, 5-1-5 Kashiwa-no-ha, Kashiwa, Japan 277-8568 \\ ${ }^{3}$ Department of Physics, California Institute of Technology, Pasadena, California 91125, USA \\ ${ }^{4}$ Michigan Center for Theoretical Physics, Physics Department, University of Michigan, Ann Arbor, Michigan 48109, USA \\ ${ }^{5}$ Center for Particle Astrophysics, Fermi National Accelerator Laboratory, Batavia, Illinois 60510, USA \\ ${ }^{6}$ University of California, Santa Cruz, Physics Department, Santa Cruz, California 95064, USA \\ ${ }^{7}$ Theoretical Physics Group, LBNL, Berkeley, California 94720, USA
}

(Received 18 December 2009; published 2 February 2010)

\begin{abstract}
Dark matter decaying or annihilating into $\mu^{+} \mu^{-}$or $\tau^{+} \tau^{-}$has been proposed as an explanation for the $e^{ \pm}$anomalies reported by PAMELA and Fermi. Recent analyses show that IceCube, supplemented by DeepCore, will be able to significantly constrain the parameter space of decays to $\mu^{+} \mu^{-}$, and rule out decays to $\tau^{+} \tau^{-}$and annihilations to $\mu^{+} \mu^{-}$in less than five years of running. These analyses rely on measuring tracklike events in IceCube + DeepCore from down-going $\nu_{\mu}$. In this paper we show that by instead measuring cascade events, which are induced by all neutrino flavors, IceCube + DeepCore can rule out decays to $\mu^{+} \mu^{-}$in only three years of running, and rule out decays to $\tau^{+} \tau^{-}$and annihilation to $\mu^{+} \mu^{-}$in only one year of running. These constraints are highly robust to the choice of dark matter halo profile and independent of dark matter-nucleon crosssection.
\end{abstract}

DOI: 10.1103/PhysRevD.81.043508

PACS numbers: 95.35.+d, 14.60.Pq, 98.80.Cq

\section{INTRODUCTION}

The existence of dark matter has been established by numerous observations. Although it constitutes most of the matter in the universe [1], the nature of dark matter remains largely unknown. One widely held possibility is that it is a new fundamental particle produced in the early universe and present today as a thermal relic. Among the best motivated of these are the so-called weakly interacting massive particles, or "WIMPs" (for reviews, see Refs. [2,3]) which are predicted to be undergoing annihilations [4-7] and possibly decays [8] in the current epoch.

Recently, the instruments PAMELA [9], ATIC [10], PPB-BETS [11], and Fermi [12] have observed features in the cosmic-ray $e^{ \pm}$spectrum and a positron fraction that are inconsistent with known backgrounds. While these anomalies may be due to unidentified astrophysical sources [13], one exciting possibility is that they are due to the decay [14-20] or annihilation [21-26] of dark matter particles into standard model states. Even in the case that anomalies are not caused by new physics in the dark sector, the constraints are generally applicable to dark matter models.

In order for dark matter to explain the anomalies, the products of decay or annihilation must be primarily leptonic. In either case, the Fermi observation gives the most precise preferred region in mass and lifetime/cross section; for decays, only $\mu^{+} \mu^{-}$and $\tau^{+} \tau^{-}$final states fit the data, while for annihilations a small region of $e^{+} e^{-}$is also permitted [27]. For the allowed parameter space, decays or annihilations into hadrons and weak and Higgs bosons are severely limited. Decays are constrained by the Fermi observation of the isotropic diffuse gamma-ray flux [28], and annihilations are constrained by the production of antiprotons [29].

Explaining the anomalies with annihilations has an additional challenge. In order to match the observed rates, the cross section required is $10^{3}-10^{4}$ times that expected for thermal production of the dark matter in the early universe. This necessitates nonthermal production mechanisms or low-velocity enhancements to the cross section, such as the Sommerfeld enhancement $[24,26]$ or the Breit-Wigner enhancement [30,31]. Moreover, high cross-section annihilations to leptonic states are tightly constrained by synchrotron radio emissions from the galactic center, although this constraint can be evaded if the true galactic dark matter halo profile is much less steep at the galactic center than benchmark profiles $[27,32]$.

For either decays or annihilations, neutrino observations may provide the strongest constraints-or the most promising corroborating signatures-for dark matter to be the source of the anomalies. With the exception of the disfavored $e^{+} e^{-}$channel, the required leptonic final states decay into neutrinos. These travel undeflected from their sources, eliminating any uncertainties in modeling their propagation. Moreover, astrophysical sources that may explain the anomalies are not expected to produce a large flux of neutrinos. Pulsars, for example, would generate at most $\mathcal{O}(1)$ events/year at a full $\mathrm{km}$-scale detector, and only at energies greater than $10 \mathrm{TeV}$ [33].

There are, however, challenges to constraining dark matter models with neutrinos. As they are observed only by collecting Cherenkov light from induced particle show- 
ers or from secondary muons, angular resolution is poor compared to gamma-ray observatories. Also, there are significant backgrounds from atmospheric muon and neutrino fluxes. However, if these backgrounds can be controlled, the poor angular resolution need not be a barrier; indeed by integrating the flux over a large area of the sky, the resulting constraint is much less sensitive to the choice of dark matter profile [34,35]. Moreover, by observing the galactic and extragalactic diffuse dark matter, rather than any that may have been captured by the Sun or the Earth, any constraints will be independent of the dark matternucleon cross sections, which are related to final states in a model-specific way [36].

Recent analyses show the power of neutrino constraints, using various strategies to reduce the effect of atmospheric backgrounds. The Super-Kamiokande observatory resides in the northern hemisphere, facing away from the galactic center, minimizing atmospheric backgrounds. Measurements of upward-going muons place a limit on the flux of galactic $\nu_{\mu}$, providing a robust constraint that eliminates annihilations to $\tau^{+} \tau^{-}$as a source of the $e^{ \pm}$anomalies $[27,37]$. The IceCube observatory, on the other hand, resides at the South Pole where down-going atmospheric fluxes are coincident with the neutrinos from the galactic center. The overwhelming background of atmospheric muons can be suppressed by event selection to establish an isotropic diffuse flux limit [38-40], but this limit only starts at a high energy threshold $\mathcal{O}(10 \mathrm{TeV})$, and yields a relatively weak constraint as we will show.

Currently under construction is DeepCore [41], an in-fill of the IceCube detector which will use the outer instrumented volume as a veto on downward-going muons to a level of one part in $10^{6}$ [42]. This will allow the galactic neutrino flux to be measured and compared against the atmospheric neutrino flux, providing a constraint on dark matter decays and annihilations. Recent work $[43,44]$ by some of the authors shows that IceCube + DeepCore will be able to significantly constrain the parameter space of decays to $\mu^{+} \mu^{-}$, and rule out decays to $\tau^{+} \tau^{-}$and annihilation to $\mu^{+} \mu^{-}$as possible sources of the anomalies in less than five years of running.

Recently, IceCube + DeepCore has demonstrated in simulations the ability to distinguish between tracklike events, which are due to the charged-current interactions of $\nu_{\mu}$, and cascade events, which are induced by $\nu_{e, \tau}$ through charged-current interactions and by all neutrino flavors through neutral-current interactions $[45,46]$. This is very useful for constraining dark matter neutrino fluxes because $\nu_{\mu}$ is the dominant flavor of atmospheric neutrinos above $40 \mathrm{GeV}[47,48]$. The neutrino-nucleon cross sections are the same for all flavors, so signal would be considerably enhanced, while background would be reduced because $\nu_{\mu}$ only creates cascade events through the neutral-current interaction, which is lower in cross section than the charged-current interaction $[34,49,50]$

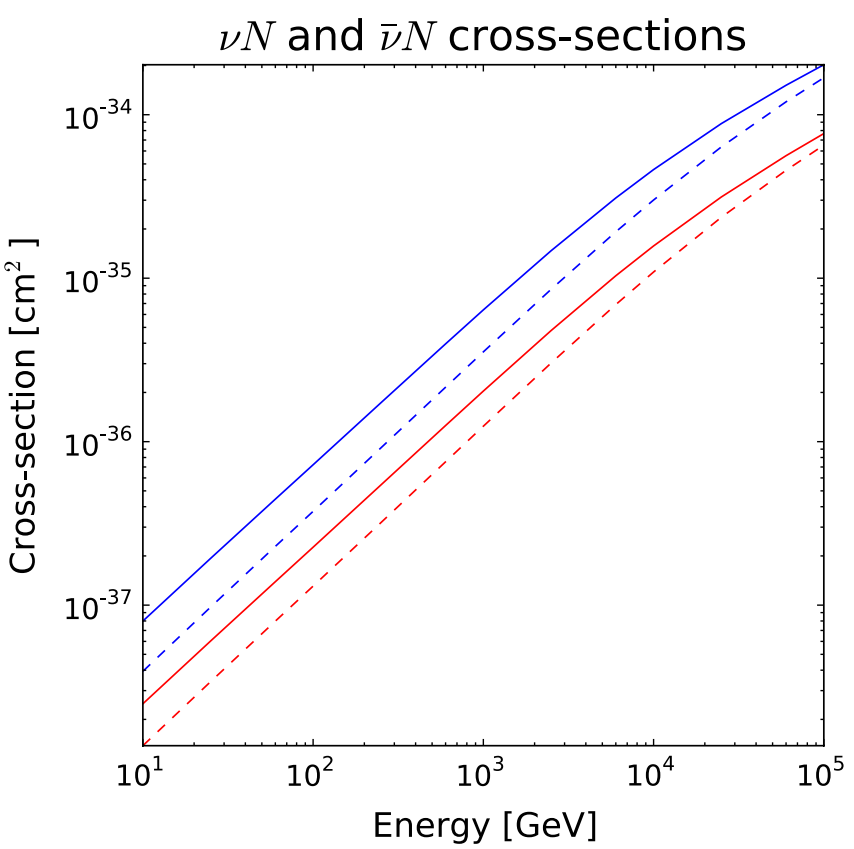

FIG. 1 (color online). Cross sections for (anti)neutrinonucleon interactions, given by Ref. [49]. The blue lines are for charged-current interactions, and the red lines are for neutralcurrent interactions. Solid lines are for neutrinos and dashed lines are for antineutrinos.

(see Fig. 1). Moreover, cascade events are easy to distinguish from the tracks caused by any atmospheric muons that are not vetoed by the outer volume.

In this paper we show that observation of cascade events at IceCube + DeepCore can enhance the neutrino constraints on dark matter, and rule out (or corroborate) dark matter decays to $\mu^{+} \mu^{-}$or $\tau^{+} \tau^{-}$and annihilation to $\mu^{+} \mu^{-}$as sources of the observed $e^{ \pm}$cosmic-ray anomalies in a much shorter time compared to searches that rely solely on tracklike events.

\section{ANALYSIS}

For brevity, in the expressions below we write only the terms for neutrinos and not the terms for antineutrinos. The terms are identical, except replacing " $\nu$ " by " $\nu$ ".

\section{A. Galactic flux signal and background}

The flux of neutrinos from the galactic dark matter halo is given by

$$
\frac{d \Phi_{\nu_{i}}}{d E d \Omega}=\frac{1}{4 \pi}\left(r_{\odot} \rho_{\odot}\right) \frac{1}{m \tau} \frac{d N_{\nu_{i}}}{d E} J_{1}(\Delta \Omega)
$$

for decays and by

$$
\frac{d \Phi_{\nu_{i}}}{d E d \Omega}=\frac{1}{8 \pi}\left(r_{\odot} \rho_{\odot}^{2}\right) \frac{1}{m^{2}}\langle\sigma v\rangle \frac{d N_{\nu_{i}}}{d E} J_{2}(\Delta \Omega)
$$

for annihilations, where $r_{\odot}=8.5 \mathrm{kpc}$ is the distance from the Sun to the galactic center [51], $\rho_{\odot}=0.3 \mathrm{GeV} \mathrm{cm}^{-3}$ is 
the dark matter density in the solar neighborhood, $m$ is the dark matter mass, and $\tau$ and $\langle\sigma v\rangle$ are the dark matter lifetime and thermally averaged cross section, respectively. $J_{n}$ is the line-of-sight (los) integral through the halo profile:

$$
J_{n}(\Delta \Omega)=\frac{1}{\Delta \Omega} \int_{\Delta \Omega} d \Omega \int_{\operatorname{los}} \frac{d s}{r_{\odot}}\left(\frac{\rho}{\rho_{\odot}}\right)^{n},
$$

where $\Delta \Omega$ is the region of sky observed. In this analysis we use the Navarro-Frenk-White (NFW) profile [52]

$$
\rho(r)=\rho_{\odot}\left(\frac{r_{\odot}}{r}\right)\left(\frac{1+r_{\odot} / r_{s}}{1+r / r_{s}}\right)^{2}
$$

with $r_{s}=20 \mathrm{kpc}$.

The neutrino source spectra $d N_{\nu_{i}} / d E$ for flavors $i$ are given by PYTHIA [53] simulation. Assuming $\sin ^{2} 2 \theta_{12}: \sin ^{2} 2 \theta_{13}: \sin ^{2} 2 \theta_{23} \approx 1: 1: 0$, the flavor distribution will be $1: 1: 1$ as the neutrinos reach the Earth, having traveled a variety of long distances across the galaxy and being well mixed through vacuum oscillations.

As discussed in the Introduction, the only background to cascade events is atmospheric neutrinos after the veto suppresses the background of atmospheric muons to one part in $10^{6}$ and event selection is used to eliminate the rest. The $\nu_{\mu}$ and $\nu_{e}$ fluxes are given by Ref. [47], where the $\nu_{\mu}$ flux agrees well with AMANDA observation [54]. At low zenith angles, the flux of background $\nu_{\mu}$ is $\sim 20$ times greater than the flux of $\nu_{e}$ from $40 \mathrm{GeV}$ to $100 \mathrm{TeV}$; it is $\sim 1000$ times greater than the flux of $\nu_{\tau}$ (see Ref. [48]). However, below $40 \mathrm{GeV}$, especially at high zenith angles, the atmospheric fluxes of the three flavors only differ by $\mathcal{O}(1)$ due to flavor mixing. Since modeling the background (and signal) below $40 \mathrm{GeV}$ would require simulating flavor mixing as the neutrinos propagate through the limb of the Earth, for this analysis we simply set an energy cutoff of $E_{\text {thresh }}=40 \mathrm{GeV}$.

To obtain the event rates due to the galactic and background fluxes, we first set energy bins for dark matter of mass $m$ to be $\left[\max \left(E_{\text {thresh }}, m / 10\right), m / 2\right]$ for decays and $\left[\max \left(E_{\text {thresh }}, m / 5\right), m\right]$ for annihilations. Note that the bin width is much greater than the energy resolution, $\log _{10}\left(E_{\max } / E_{\min }\right) \simeq 0.4$ for tracklike events and $\log _{10}\left(E_{\max } / E_{\min }\right) \simeq 0.18$ for cascade events [42]. We then integrate the flux times the effective area over the energy for each bin.

For tracklike events due to $\nu_{\mu}$, the event rate is

$$
\Gamma_{\mathrm{tr}}=\int d \Omega \int_{E_{\min }}^{E_{\max }} d E \rho_{\mathrm{ice}} N_{A} V_{\mathrm{tr}}\left(\left[\sigma_{\nu N}(E)\right]_{\mathrm{CC}} \frac{d \Phi_{\nu_{\mu}}}{d E d \Omega}\right)
$$

where $\rho_{\text {ice }}=0.9 \mathrm{~g} \mathrm{~cm}^{-3}$ is the density of ice, $N_{A}=$ $6.022 \times 10^{23} \mathrm{~g}^{-1}$ is Avogadro's number (to convert mass to number of nucleons), $\left[\sigma_{\nu N}(E)\right]_{\mathrm{CC}}$ is the neutrinonucleon cross section for the charged-current interaction, and $V_{\text {tr. }} \approx 0.04 \mathrm{~km}^{3}$ is the effective volume of the detector for tracklike events [42]. Note that we do not add the residual atmospheric muon background to the background of tracklike events due to the uncertainty in its value.

For cascade events we use the instrumented volume $V_{\text {casc }} \approx 0.02 \mathrm{~km}^{3}[42,55]$, the charged-current interaction for $\nu_{e, \tau}$, and the neutral-current interaction for all flavors to obtain

$$
\begin{aligned}
\Gamma_{\mathrm{casc}}= & \int d \Omega \int_{E_{\min }}^{E_{\max }} d E \rho_{\mathrm{ice}} N_{A} V_{\text {casc }} \\
& \times\left(\left[\sigma_{\nu N}(E)\right]_{\mathrm{CC}} \frac{d \Phi_{\nu_{e, \tau}}}{d E d \Omega}+\left[\sigma_{\nu N}(E)\right]_{\mathrm{NC}} \frac{d \Phi_{\nu_{e, \mu, \tau}}}{d E d \Omega}\right)
\end{aligned}
$$

Unlike tracklike events, cascade events are well contained, so the effective volume for their detection will vary little with energy. Also, we assume that in neutral-current interactions all the energy of the neutrino goes into the cascade. Taking partial energy transfer into account would yield a modest improvement in significance, since most of the signal is from $\nu_{e, \tau}$ charged-current interactions but most of the background is from $\nu_{\mu}$ neutral-current interactions. ${ }^{1}$

Finally, because the pointing capability for cascades is approximately $50^{\circ}[42,45]$ and the pointing capability for tracklike events has yet to be established, we integrate over the $2 \pi$ field of view around the galactic center. As mentioned before, this should provide a constraint which is robust to changes in dark matter halo profile. Specifically, the fractional change between the NFW profile used here and the much less steep isothermal profile for $J_{1}(2 \pi)$ is $\mathcal{O}\left(10^{-3}\right)$, and for $J_{2}(2 \pi)$ is $\mathcal{O}(0.1)$.

\section{B. Extragalactic isotropic diffuse flux}

For comparison to the DeepCore constraints from downgoing fluxes, we calculate the constraint from the isotropic diffuse flux limit for AMANDA-II from tracklike events [39] and the projected limit for IceCube from cascade events [40].

The main contribution from dark matter decay to the isotropic diffuse flux is that from extragalactic dark matter residing on cosmological scales. The flux from the decay of cosmological dark matter is given by the previously derived [28,57] formula

$$
\begin{aligned}
\left(\frac{d \Phi_{\nu_{i}}}{d E d \Omega}\right)^{(\text {ex. })}= & \frac{c}{4 \pi} \frac{\Omega_{\mathrm{DM}} \rho_{c}}{H_{0} \Omega_{\mathrm{M}}^{1 / 2}} \frac{1}{m \tau} \int_{1}^{\infty} d y \frac{y^{-3 / 2}}{\sqrt{1+\Omega_{\Lambda} / \Omega_{\mathrm{M}} y^{-3}}} \\
& \times\left(\frac{d N_{\nu_{i}}}{d(E y)}\right)
\end{aligned}
$$

where $y \equiv 1+z$, with $z$ being the redshift, $H_{0}=$ $71.9 \mathrm{~km} \mathrm{~s}^{-1} \mathrm{Mpc}^{-1}$ is the Hubble constant, $\rho_{c}=$ $3 H_{0}^{2} /\left(8 \pi G_{N}\right)$ is the critical density, $\Omega_{\mathrm{M}}=0.258, \Omega_{\mathrm{DM}}=$ 0.214 , and $\Omega_{\Lambda}=0.721$ are, respectively, the total matter,

\footnotetext{
${ }^{1}$ Inelasticity curves are given in Ref. [56], but only for energies $10 \mathrm{TeV}$ and higher.
} 
dark matter, and dark energy densities divided by the critical density [1]. The isotropic diffuse flux from the annihilations of cosmological dark matter is too small to be relevant, since the density of dark matter on cosmological scales is very low, and the flux is suppressed by another power of $\rho_{c} / m$.

Because of the loss of signal due to event selection and the presence of background fluxes, both the AMANDA-II limit and the projected IceCube limit are only valid at energies greater than $\sim 20 \mathrm{TeV}$. Below these thresholds we add the atmospheric background flux to these limits, and use these total fluxes to calculate the constraints on the dark matter lifetime.

\section{RESULTS}

The results for dark matter decays are shown in Fig. 2; the regions below the contours are excluded. The black contour ("Super-K up- $\mu$ ") is the Super-Kamiokande limit to $3 \sigma$ from up-going muons discussed in the Introduction. The orange band is the preferred region to fit the PAMELA $e^{ \pm}$anomaly, and the red ellipses are the preferred region to fit the Fermi $e^{ \pm}$anomaly. These three regions are given by Ref. [27] up to mass $30 \mathrm{TeV}$ and lifetime $10^{27} \mathrm{~s}$.
The dashed green line ("AMANDA-II diff.") is the constraint to $90 \%$ confidence from the AMANDA-II limit on the isotropic diffuse flux of $\nu_{\mu}$, and the solid green line ("IceCube casc. diff.") is the constraint to $90 \%$ confidence from the projected IceCube limit on the isotropic diffuse flux using cascade events. Since the flux from cosmological dark matter is weak to begin with, contributing only $1 \%$ of the total flux over the $2 \pi$ facing the galactic center, we see that it is quickly overwhelmed by the atmospheric flux below $\sim 40 \mathrm{TeV}$. Nonetheless, due to the lower background of atmospheric $\nu_{e}$ compared to $\nu_{\mu}$ at these high energies, using cascade events improves the constraint by a factor of $\sim 5$.

The dashed blue line ("DeepCore tr. 5yr") is the constraint to $2 \sigma$ from IceCube + DeepCore for tracklike events after five years of running, and the solid blue lines are the constraints to $2 \sigma$ for cascade events after one year ("DeepCore casc. 1yr") and three years ("DeepCore casc. 3yr") of running. We see that for the $\mu^{+} \mu^{-}$final state, while tracklike events can only reduce the available Fermi-preferred parameter space in five years, cascade events can rule it out altogether in only three years. Similarly for the $\tau^{+} \tau^{-}$final state, tracklike events can rule out the parameter space in less than five years, but
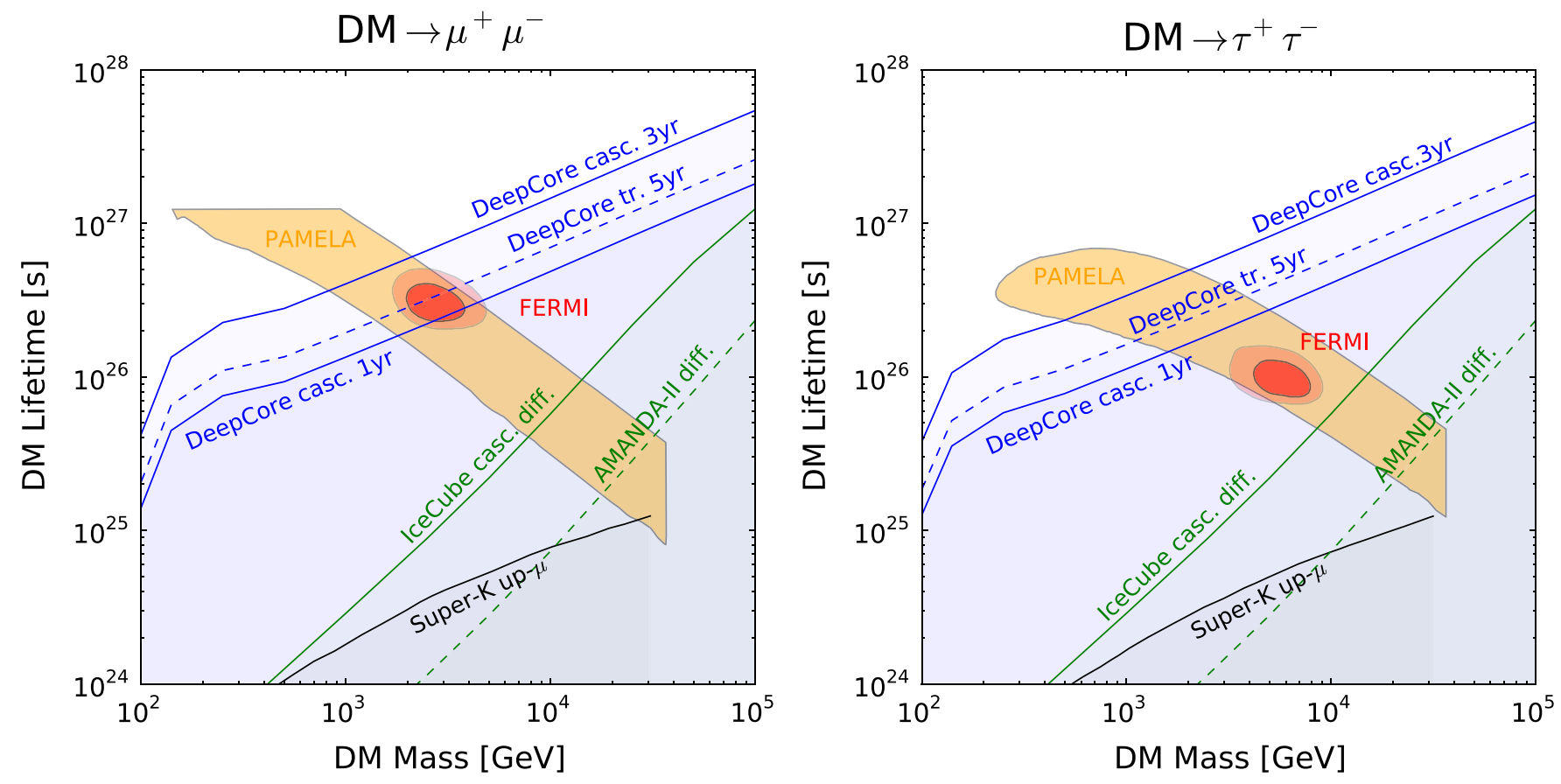

FIG. 2 (color online). Constraints for decay to $\mu^{+} \mu^{-}$(left) and $\tau^{+} \tau^{-}$(right); the regions below the contours are excluded. The black contour ("Super-K up- $\mu$ ") is the Super-Kamiokande limit to $3 \sigma$ from up-going muons, the orange band is the PAMELA-preferred region, and the red ellipses are the Fermi-preferred region; these three are given by Ref. [27]. The dashed green line ("AMANDA-II diff.") is the constraint to $90 \%$ confidence from the AMANDA-II limit on the isotropic diffuse flux of $\nu_{\mu}$, and the solid green line ("IceCube casc. diff.") is the constraint to $90 \%$ confidence from the projected IceCube limit on the isotropic diffuse flux using cascade events. The dashed blue line ("DeepCore tr. 5yr") is the constraint to $2 \sigma$ from IceCube + DeepCore for $\nu_{\mu}$ tracklike events after five years of running, and the solid blue lines are the constraints to $2 \sigma$ for all-flavor cascade events after one year ("DeepCore casc. 1yr") and three years ("DeepCore casc. 3yr") of running. 

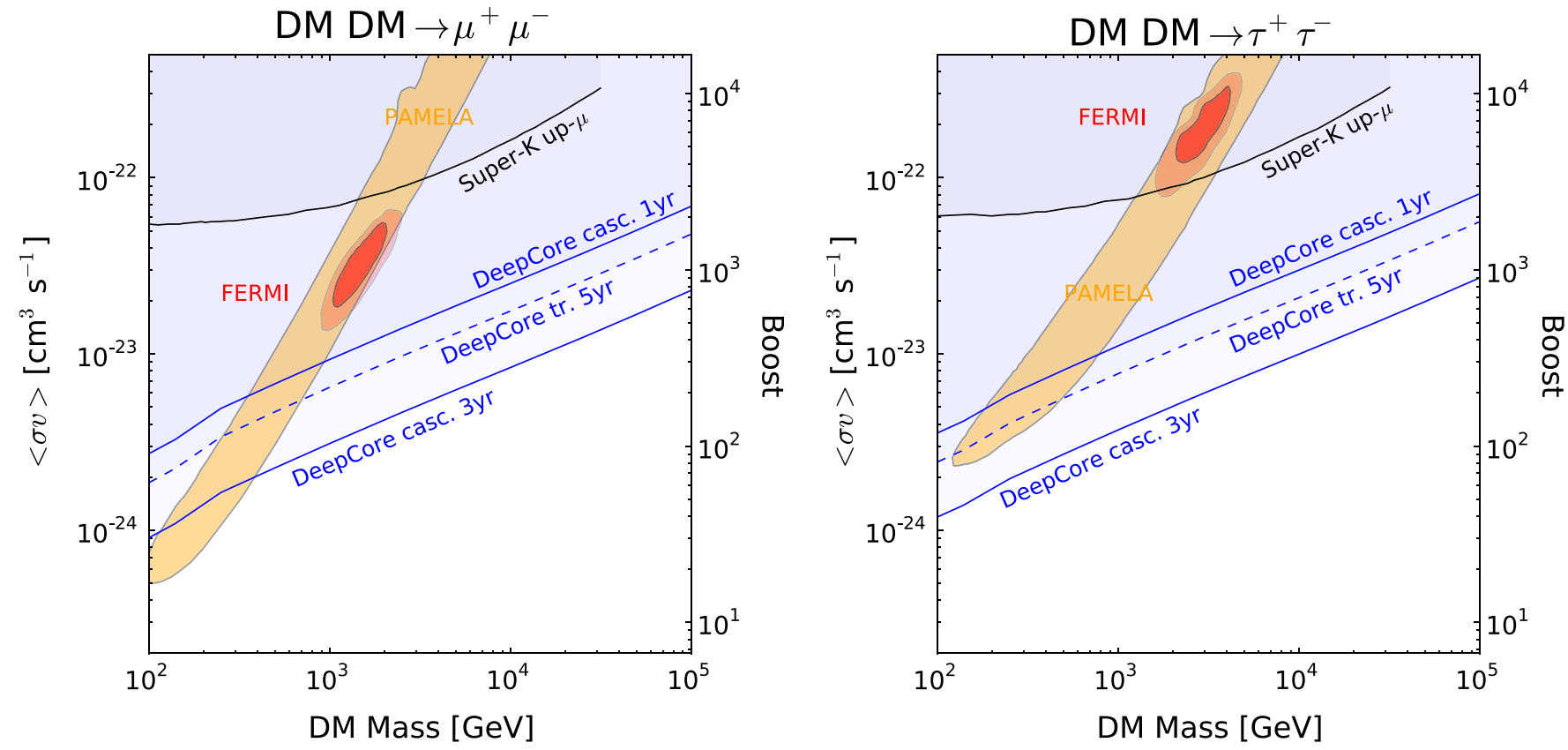

FIG. 3 (color online). Constraints for annihilation to $\mu^{+} \mu^{-}$(left) and $\tau^{+} \tau^{-}$(right); the regions above the contours are excluded. The black contour ("Super-K up- $\mu$ ") is the Super-Kamiokande limit to $3 \sigma$ from up-going muons, the orange band is the PAMELApreferred region, and the red ellipses are the Fermi-preferred region; these three are given by Ref. [27]. The dashed blue line ("DeepCore tr. 5yr") is the constraint to $2 \sigma$ from IceCube + DeepCore for $\nu_{\mu}$ tracklike events after five years of running, and the solid blue lines are the constraints to $2 \sigma$ for all-flavor cascade events after one year ("DeepCore casc. 1yr") and three years ("DeepCore casc. 3yr") of running.

with cascade events it will only take one year. Note the weakening of the constraints below $m=250 \mathrm{GeV}$, where the energy per final-state particle is less than $125 \mathrm{GeV}$. This is caused by the energy cutoff at $40 \mathrm{GeV}$.

The results for annihilation are shown in Fig. 3; the regions above the contours are excluded. The plots show the same constraints as for decay, except that no isotropic limits are shown because they are weaker than the SuperKamiokande limit by a factor $\sim 10^{5}$ due to the low density of dark matter on cosmological scales. As with decays, cascade events greatly accelerate the development of a useful constraint. For the $\mu^{+} \mu^{-}$final state the region by the Fermi data can be eliminated in only one year.

The exclusion plot for annihilations to $\tau^{+} \tau^{-}$is shown only for completeness, as the Fermi-preferred region has already been eliminated by the Super-Kamiokande observation of upward-going muons. However, it may provide a useful generic constraint on all dark matter models irrespective of the $e^{ \pm}$anomalies.

\section{CONCLUSION}

We have shown that, by using cascade events, IceCube + DeepCore can more quickly establish constraints on dark matter models that would explain the reported $e^{ \pm}$anomalies, and over time establish stronger constraints than from tracklike events. Specifically, tracklike events will be able to significantly constrain the parameter space of decays to $\mu^{+} \mu^{-}$, and rule out decays to $\tau^{+} \tau^{-}$and annihilations to $\mu^{+} \mu^{-}$in less than five years of running. In comparison, cascade events can rule out decays to $\mu^{+} \mu^{-}$in only three years, and rule out decays to $\tau^{+} \tau^{-}$and annihilation to $\mu^{+} \mu^{-}$after only one year. Moreover, these constraints are highly robust to the choice of dark matter halo profile and independent of dark matter-nucleon cross section.

In closing, we note two interesting possibilities for future work. First, if the pointing accuracy for tracklike events at IceCube + DeepCore is established to be less than $10^{\circ}$, the signal-to-noise for annihilations may be significantly enhanced by observing a smaller region around the galactic center, possibly outperforming cascade searches (albeit with greater dependence on the choice of dark matter halo profile). This would strengthen the discovery potential for dark matter because the galactic center could be identified as a localized source of excess neutrinos. Second, because the $\nu_{\tau}$ atmospheric background is so low at energies above $40 \mathrm{GeV}$ and at low zenith angles, if IceCube + DeepCore can demonstrate efficient $\nu_{\tau}$ discrimination [46], signal to noise could be increased by a factor $\sim 100$. This would put leptophilic dark matter to a severe test.

\section{ACKNOWLEDGMENTS}

The authors would like to thank D. Grant, D. J. Koskinen, and I. Taboada for answering questions about 
DeepCore. S. K. M. is supported by World Premier International Research Center Initiative (WPI Initiative), MEXT, Japan, and would also like to thank G. Lambard for useful discussions. M.R.B. is supported by the U.S. Department of Energy, under Grant No. DE-FG03-92ER40701. K.F. is supported by the U.S. Department of Energy and MCTP via the University of Michigan. D. S. is supported by NSF Grant No. AST-0507117 and GAANN (D. S.). H. M. is supported in part by World Premier International Research Center Initiative (WPI Initiative), MEXT, Japan, in part by the U.S. DOE under Contract No. DE-AC03-76SF00098, and in part by the NSF under Grant No. PHY-04-57315.
[1] J. Dunkley et al., Astrophys. J. Suppl. Ser. 180, 306 (2009).

[2] G. Jungman, M. Kamionkowski, and K. Griest, Phys. Rep. 267, 195 (1996).

[3] G. Bertone, D. Hooper, and J. Silk, Phys. Rep. 405, 279 (2005).

[4] M. Srednicki, K. A. Olive, and J. Silk, Nucl. Phys. B279, 804 (1987).

[5] K. Freese, Phys. Lett. 167B, 295 (1986); L. M. Krauss, M. Srednicki, and F. Wilczek, Phys. Rev. D 33, 2079 (1986).

[6] J. R. Ellis, R. A. Flores, K. Freese, S. Ritz, D. Seckel, and J. Silk, Phys. Lett. B 214, 403 (1988); M. S. Turner and F. Wilczek, Phys. Rev. D 42, 1001 (1990); M. Kamionkowski and M. S. Turner, Phys. Rev. D 43, 1774 (1991); J. Silk and M. Srednicki, Phys. Rev. Lett. 53, 624 (1984); L. Bergstrom, P. Ullio, and J.H. Buckley, Astropart. Phys. 9, 137 (1998).

[7] N. W. Evans, F. Ferrer, and S. Sarkar, Phys. Rev. D 69, 123501 (2004); L. Bergstrom and D. Hooper, Phys. Rev. D 73, 063510 (2006).

[8] P. Gondolo, Phys. Lett. B 295, 104 (1992).

[9] O. Adriani et al. (PAMELA Collaboration), Nature (London) 458, 607 (2009).

[10] J. Chang et al. (ATIC Collaboration), Nature (London) 456, 362 (2008).

[11] S. Torii et al., arXiv:0809.0760.

[12] A. A. Abdo et al. (The Fermi LAT Collaboration), Phys. Rev. Lett. 102, 181101 (2009).

[13] D. Hooper, P. Blasi, and P.D. Serpico, J. Cosmol. Astropart. Phys. 01 (2009) 025; S. Profumo, arXiv:0812.4457.

[14] C. R. Chen, F. Takahashi, and T. T. Yanagida, Phys. Lett. B 671, 71 (2009); 673, 255 (2009); C. R. Chen, M. M. Nojiri, F. Takahashi, and T. T. Yanagida, Prog. Theor. Phys. 122, 553 (2009).

[15] C. R. Chen and F. Takahashi, J. Cosmol. Astropart. Phys. 02 (2009) 004.

[16] P. F. Yin, Q. Yuan, J. Liu, J. Zhang, X. J. Bi, S. H. Zhu, and X. Zhang, Phys. Rev. D 79, 023512 (2009).

[17] K. Ishiwata, S. Matsumoto, and T. Moroi, Phys. Lett. B 675, 446 (2009).

[18] S. Shirai, F. Takahashi, and T. T. Yanagida, Phys. Lett. B 675, 73 (2009); arXiv:0902.4770.

[19] A. Ibarra, A. Ringwald, D. Tran, and C. Weniger, J. Cosmol. Astropart. Phys. 08 (2009) 017; see also A. Ibarra, A. Ringwald, and C. Weniger, J. Cosmol. Astropart. Phys. 01 (2009) 003.
[20] M. Ibe, H. Murayama, S. Shirai, and T. T. Yanagida, J. High Energy Phys. 11 (2009) 120.

[21] V. Barger, W. Y. Keun, D. Marfatia, and G. Shaughnessy, Phys. Lett. B 672, 141 (2009).

[22] I. Cholis, L. Goodenough, D. Hooper, M. Simet, and N. Weiner, Phys. Rev. D 80, 123511 (2009).

[23] L. Bergstrom, J. Edsjo, and G. Zaharijas, Phys. Rev. Lett. 103, 031103 (2009).

[24] A. Sommerfeld, Ann. Phys. (Leipzig) 403, 257 (1931); M. Cirelli and A. Strumia, arXiv:0808.3867; M. Cirelli, M. Kadastik, M. Raidal, and A. Strumia, Nucl. Phys. B813, 1 (2009).

[25] R. Harnik and G. D. Kribs, Phys. Rev. D 79, 095007 (2009); A. E. Nelson and C. Spitzer, arXiv:0810.5167; I. Cholis, D. P. Finkbeiner, L. Goodenough, and N. Weiner, J. Cosmol. Astropart. Phys. 12 (2009) 007; K. M. Zurek, Phys. Rev. D 79, 115002 (2009); P. J. Fox and E. Poppitz, Phys. Rev. D 79, 083528 (2009); I. Cholis, G. Dobler, D. P. Finkbeiner, L. Goodenough, and N. Weiner, Phys. Rev. D 80, 123518 (2009); S. C. Park and J. Shu, Phys. Rev. D 79, 091702(R) (2009); D. Hooper and K. M. Zurek, Phys. Rev. D 79, 103529 (2009); D. Hooper and T. M. P. Tait, Phys. Rev. D 80, 055028 (2009).

[26] N. Arkani-Hamed, D. P. Finkbeiner, T. R. Slatyer, and N. Weiner, Phys. Rev. D 79, 015014 (2009).

[27] P. Meade, M. Papucci, A. Strumia, and T. Volansky, arXiv:0905.0480.

[28] C.-R. Chen, S. K. Mandal, and F. Takahashi, arXiv:0910.2639.

[29] O. Adriani et al., Phys. Rev. Lett. 102, 051101 (2009).

[30] D. Feldman, Z. Liu, and P. Nath, Phys. Rev. D 79, 063509 (2009).

[31] M. Ibe, H. Murayama, and T. T. Yanagida, Phys. Rev. D 79, 095009 (2009).

[32] G. Bertone, M. Cirelli, A. Strumia, and M. Taoso, J. Cosmol. Astropart. Phys. 03 (2009) 009.

[33] A. Bhadra and R. K. Dey, Mon. Not. R. Astron. Soc. 395, 1371 (2009); W. Bednarek, Phys. Rev. D 79, 123010 (2009).

[34] J. F. Beacom, N. F. Bell, and G. D. Mack, Phys. Rev. Lett. 99, 231301 (2007).

[35] H. Yuksel, S. Horiuchi, J. F. Beacom, and S. Ando, Phys. Rev. D 76, 123506 (2007).

[36] A. Gould, Astrophys. J. 388, 338 (1992); G. Jungman, M. Kamionkowski, and K. Griest, Phys. Rep. 267, 195 (1996); D. Hooper and J. Silk, New J. Phys. 6, 23 (2004). 
[37] S. Palomares-Ruiz, Phys. Lett. B 665, 50 (2008); J. Hisano, M. Kawasaki, K. Kohri, and K. Nakayama, Phys. Rev. D 79, 043516 (2009).

[38] M. Kowalski, J. Cosmol. Astropart. Phys. 05 (2005) 010.

[39] L. Gerhardt (IceCube Collaboration), in Proceedings of the 30th ICRC (2007).

[40] J. Kiryluk (IceCube Collaboration), in Proceedings of the 31st ICRC (2009).

[41] S. R. Klein (IceCube Collaboration), IEEE Trans. Nucl. Sci. 56, 1141 (2009).

[42] E. Resconi (IceCube Collaboration), Nucl. Instrum. Methods Phys. Res., Sect. A 602, 7 (2009).

[43] D. Spolyar, M. Buckley, K. Freese, D. Hooper, and H. Murayama, arXiv:0905.4764.

[44] M. R. Buckley, K. Freese, D. Hooper, D. Spolyar, and H. Murayama, arXiv:0907.2385.

[45] E. Middell, J. McCartin, and M. D'Agostino (IceCube Collaboration), in Proceedings of the 31st ICRC (2009).

[46] D. Grant, D. J. Koskinen, and C. Rott (IceCube Collaboration), in Proceedings of the 31st ICRC (2009).

[47] M. Honda, T. Kajita, K. Kasahara, S. Midorikawa, and T.
Sanuki, Phys. Rev. D 75, 043006 (2007); J. G. Learned and K. Mannheim, Annu. Rev. Nucl. Part. Sci. 50, 679 (2000).

[48] F.-F. Lee and G.-L. Lin, Astropart. Phys. 25, 64 (2006).

[49] R. Gandhi, C. Quigg, M. H. Reno, and I. Sarcevic, Phys. Rev. D 58, 093009 (1998).

[50] J. F. Beacom and J. Candia, J. Cosmol. Astropart. Phys. 11 (2004) 009.

[51] R. Catena and P. Ullio, arXiv:0907.0018.

[52] J. F. Navarro, C. S. Frenk, and S. D. M. White, Astrophys. J. 490, 493 (1997).

[53] T. Sjostrand, S. Mrenna, and P. Skands, J. High Energy Phys. 05 (2006) 026.

[54] R. Abbasi et al. (IceCube Collaboration), Phys. Rev. D 79, 102005 (2009).

[55] A. B. McDonald, C. Spiering, S. Schoenert, E. T. Kearns, and T. Kajita, Rev. Sci. Instrum. 75, 293 (2004).

[56] R. Gandhi, C. Quigg, M.H. Reno, and I. Sarcevic, Astropart. Phys. 5, 81 (1996).

[57] A. Ibarra and D. Tran, Phys. Rev. Lett. 100, 061301 (2008). 